\title{
Optimal prize allocations in group contests
}

\author{
Francesco Trevisan ${ }^{1}$
}

Received: 29 March 2019 / Accepted: 5 March 2020 / Published online: 20 March 2020

(c) The Author(s) 2020

\begin{abstract}
We characterize the optimal prize allocation, namely the allocation that maximizes a group's effectiveness, in a model of contests. The model has the following features: (i) it allows for heterogeneity between and within groups; (ii) it classifies contests as "easy" and "hard" depending on whether the marginal costs are concave or convex. Thus, we show that in an "easy" contest the optimal prize allocation assigns the entire prize to one group member, the most skilled one. Conversely, all group members receive a positive share of the prize when the contest is "hard" and players have unbounded above marginal productivities. If the contest is "hard" and the marginal productivities are bounded above, then only the most skilled group members are certain of receiving a positive share of the prize for any distribution of abilities. Finally, we study the effects of a change in the distribution of abilities within a group. Our analysis shows that if the contest is either "easy" or a particular subset of "hard", then the more the heterogeneity within a group, the higher its probability of winning the prize.
\end{abstract}

\section{Introduction}

"Soldiers generally win battles; generals get credit for them."1

Contests are ubiquitous in that they arise, for example, in wars, sports, electoral campaigns and workplace competitions. When contests arise between groups,

\footnotetext{
${ }^{1}$ Napoleon (1769-1821).
}

\begin{abstract}
I would like to thank Ed Hopkins, Santiago Sanchez Pages, Tatiana Kornienko, Jozsef Sakovics, Ina Taneva, Andrew Clausen, Marco LiCalzi, Alessandro Spiganti; audiences at the 2016 CBESS Conference on Contests: Theory and Evidence in Norwich; and the 2016 International Conference on Game Theory at Stony Brook University. This work was supported by the Economic and Social Research Council grant number ES/J500136/1.
\end{abstract}

Francesco Trevisan

f.trevisan@ed.ac.uk

1 The University of Edinburgh, Edinburgh, UK 
respective members win or lose the prize collectively. However, individuals in a group may have different positions, skills, prize valuations, and various impacts over the outcome of the competition. When the prize has private characteristics, this within group heterogeneity can lead to personalized incentives, such as highly skilled players receiving a higher share of the prize over lower skilled ones. Conversely, an egalitarian allocation of prizes may be used to encourage cooperative behaviours among members. At the beginning of the Republic of Rome, for example, the tribunes equally distributed the spoils of war among all army members, including those who only guarded the settlements and protected the wounded. After 407 $\mathrm{BC}$, the Roman Senate introduced different incentives according to the roles performed by the members of the army: ordinary soldiers received a third of the wage of the knights and half of the wage of centurions. A similar rule was used under Napoleon's Empire. Nowadays, victorious soldiers are awarded medals according to their rank in the army. Likewise, candidate prime ministers assign party members to different ministries. The assignment of the most influential people to the key ministries affects their efforts during the campaign and eventually the outcome of the elections. Finally, collective competitions are pervasive in the workplace, where, for example, department stores, retail chains, sales and production departments set up monetary rewards for the most productive teams. Nationally representative surveys reveal that $52 \%$ of firms use teamwork in the US, and $47 \%$ of British firms organized more than $90 \%$ of their workforce into teams; and $70 \%$ of Fortune 1000 companies use some form of team incentive (Bandiera et al. 2011).

In general, it seems evident that the way in which members are rewarded according to their roles and responsibilities affects how much they contribute to the group goal. Thus, the purpose of this article is twofold: to propose an allocative rule that maximizes group effectiveness, hereafter the "optimal prize allocation"; and to study how a change in the distribution of abilities within a group affects its effectiveness. We study our questions using a model with the following features: it allows for between and within groups heterogeneity; it classifies contests as "easy" and "hard" depending on whether the marginal costs are concave or convex; and each group has a manager that announces the optimal prize allocation only to her members.

Our analysis reveals the following results. In easy contests, the optimal prize allocations assigns the entire prize to one group member, the most skilled one. On the other hand, in hard contests in which players' marginal productivities are unbounded above, it rewards all group members. If the contest is hard and players' marginal productivity are bounded above, then only the most skilled members of a group are certain of receiving a positive share of the prize for any distribution of abilities. Furthermore, we find that a change in the distribution of abilities within a group affects the probability of winning of all competing groups. Specifically, if the contest is either easy or a specific subset of hard, then the more the heterogeneity within a group, the higher the group probability of winning. Finally, we rank the probability of winning of the competing groups from highest to lowest under two prize divisions: the "egalitarian" allocation, i.e. the prize is equally shared among members of the same group; and the optimal prize allocation. Surprisingly, the ranking resulting from the egalitarian allocation can be fully reversed by implementing the optimal prize allocation. 
Much progress has been made in the study of contests since the seminal work of Tullock (1980). ${ }^{2}$ In regards to group contests for public good, related set-ups are analyzed by Baik $(1993,2008)$ and Ryvkin (2011). The former shows that if players have linear cost, then only the most skilled member in every group contributes to the group cause. The latter, however, shows that all group members are active participants if costs are strictly convex. Moreover, Ryvkin (2011) studies how a contest organizer has to sort (heterogeneous) players in same size groups to maximize the aggregate effort exerted in the competition. His results are that if the players' cost function is moderately (sufficiently) steep, then a more (less) balanced competition increases aggregate effort. Thus, one could wrongly assume that the same result extends to the optimal prize allocation because both papers relate to the steepness of the cost function. However, this is not the case since the two definitions of steepness differ substantially. ${ }^{3}$ In addition, we do not focus on maximizing aggregate effort, for example by allowing groups to compete for different prizes, rather on how a group manager strategically chooses to split the prize among her members. In regards to group contests for private good, the literature has considered the following ways of prize division among the winning group members: the "egalitarian" rule, used among others, by Esteban and Ray (2001) and Cheikbossian (2012) to study the group size paradox; the "relative effort" rule, which works as an incentive device, analysed by Nitzan (1991a); and any linear combination between the "egalitarian" and the "relative effort" rule studied by Nitzan (1991b) and Nitzan and Ueda (2011, 2018), meaning part of the prize is divided equally (egalitarian rule) and the rest proportionally according to each member's effort (relative effort rule). Since the use of a relative effort allocation puts members of the same group in competition for the internal division of the prize, its full implementation eliminates the free-riding problem. However, its use effectively assumes that relative efforts can be costlessly observed and rewarded. Alternatively, a model with costs of monitoring needs to be introduced as in Ueda (2002). Even though this requirement seems innocuous, it reduces the applicability of this incentive device to few cases. ${ }^{4}$ Conversely, the egalitarian rule does not require that the individual contributions are observable, but it clearly tempts group members to free-ride on other's contributions because they win or lose the prize as a group, i.e. winning the share of the prize is a "collective good". To the best of our knowledge, the few works that study allocative schemes and do not require monitoring, assume symmetry among players and focus on the effects of within group inequality. Nitzan and Ueda (2014) focus on the effects of intragroup heterogeneity in prize shares. The authors find that in easy (hard) contests the greater (lesser) the inequality in prize share, the higher the group efficiency. Cubel and Sanchez-Pages (2014) demonstrate through Atkinson's index of inequality that egalitarian groups have a higher probability of winning the contest when the efforts

\footnotetext{
${ }^{2}$ For a review see Corchón (2007).

${ }^{3}$ For instance, all (convex) power functions are "moderately" steep in Ryvkin's model. On the other hand, we define a contest as easy or hard depending on whether the marginal costs are concave or convex.

4 See Bandiera et al. (2011) for an example about fruit picking.
} 
of the group members are complementary, or the contest is hard. There are two main differences between these models and our own: first, while they study exogenous variations in prize allocations, we analyse the endogenous choices of the optimal one; second, in our model players can be heterogeneous in their ability. If players are symmetric, group members choose the same amount of contribution in equilibrium. On the other hand, if they are heterogeneous, they react to the same incentive differently. As a matter of fact, in our model different levels of within group heterogeneity affects both the distribution of the prize among group members and the probability of winning of all groups.

Section 2 contains the preliminaries of the model; Sect. 3 presents our model of contests with managers and discusses the effect of within group heterogeneity; Sect. 4 concludes.

\section{Model preliminaries}

We first analyse a model of complete information and exogenous prize allocations so as to state an equilibrium existence result useful for Sect. 3, where we will introduce incomplete information and endogenous prize allocations. This approach is convenient both to introduce the preliminaries of our game with managers and to compare efforts under the implementation of the egalitarian rule and the optimal one. Thus, we consider a game with $N$ groups. The $i$-th group is formed by $n_{i}$ risk-neutral individuals making a total of $\sum_{i}^{N} n_{i}$ players. Players within-groups are indexed by $i k=\left(i 1, \ldots i n_{i}\right)$. All players simultaneously and irreversibly exert an effort $x_{i k} \geq 0$. The group effort is the linear sum of its members' effort, $X_{i}=\sum_{k=1}^{n_{i}} x_{i k}$. The group probability of winning is defined by the Tullock success function $\sigma_{i}=X_{i} / X$, where $X=\sum_{i=1}^{N} X_{i}$. Exerting effort is costly, but individuals are (possibly) heterogeneous in their abilities, $v_{i k} \in(0, \infty)$. The cost of effort is given by $v_{i k}^{-1} g\left(x_{i k}\right)$, and thus it is costlier for low ability individuals to exert effort. ${ }^{5}$ We impose the following assumption on $g(x)$ :

Assumption 1 (i) $g(0)=0$; (ii) $g^{\prime}(0)=0$; (iii) $g^{\prime}(x)>0$ for all $x>0$; iv) $g^{\prime \prime}(x)>0$ for all $x>0$; v) $g^{\prime \prime \prime}(x)$ exists for all $x>0$.

Part (i) states that players do not bear costs when they do not exert any effort. Part (ii) states that the marginal cost of effort at $x=0$ is zero. Part (iii) and (iv) state respectively that the effort cost function is strictly increasing and strictly convex, which ensures the existence and uniqueness of an equilibrium in which all players exert a positive effort, as long as they receive a strictly positive prize. Finally, part (v) is necessary for comparative statics. Moreover, since $g^{\prime}$ is monotonic and continuous, it has a well-defined inverse function, $f=\left(g^{\prime}\right)^{-1}$. Assumption 1 is held throughout the paper.

In our setting, the winning group is rewarded with a private good prize normalized to one, and the losing groups receive zero. For the moment, we also assume that

\footnotetext{
5 This approach to define heterogeneity is commonly used in the literature of contests, see for example Ryvkin (2011, 2013), Brookins et al. (2015) and Nitzan and Ueda (2018).
} 
the winning $i k$ member receives a share of the prize $\phi_{i k}$ according to an exogenous prize allocation $\phi_{i}=\left(\phi_{i 1}, \ldots, \phi_{i n_{i}}\right)$ s.t. $\sum_{k=1}^{n_{i}} \phi_{i k}=1$. In light of this, the player $i k$ 's expected payoff is

$$
\pi_{i k}=\frac{X_{i}}{X} \phi_{i k}-\frac{g\left(x_{i k}\right)}{v_{i k}} .
$$

Each player $i k$ 's best response to all other players' choice of effort is given by the first-order condition associated with the maximization of $\pi_{i k}$ as a function of $x_{i k}$, subject to $x_{i k} \geq 0$. Since (1) is strictly concave with respect to $x_{i k}$, the first-order condition is necessary and sufficient for the best response. It follows that the player $i k$ 's best response is

$$
\frac{X_{j \neq i}}{X^{2}} \phi_{i k} v_{i k}=g^{\prime}\left(x_{i k}\right) .
$$

As discussed in the introduction, a contest for public good and linear costs, $g\left(x_{i k}\right)=x_{i k}$, is considered in Baik (2008). The result is that in each group only the player with the lowest marginal cost exerts a positive effort. ${ }^{6}$ On the other hand, under Assumption 1, it is possible to show that there is a pure strategy Nash equilibrium in which all players that receive a positive share of the prize are active participants in equilibrium.

Lemma 1 Under Assumption 1, the contest between groups has a unique Nash equilibrium in pure strategies for any prize allocation. In equilibrium, at least one player in each group exerts a positive effort, therefore all groups exert a positive effort. The equilibrium effort $x_{i k}^{*}$ satisfies the system of Eq. (2) with equality, and defines the group i's effort as

$$
X_{i}^{*}\left(\phi_{i}\right)=\sum_{k=1}^{n_{i}} x_{i k}^{*}=\sum_{k=1}^{n_{i}} f\left(\frac{X_{j}^{*}\left(\phi_{j}\right)}{\left(X^{*}\right)^{2}} \phi_{i k} v_{i k}\right) .
$$

Finally, we define contests in the following two ways: we refer to a contest as "easy" when the cost of exerting an additional amount of effort does not rapidly increase; conversely, a contest is "hard" when an additional amount of effort leads to a significant increase in the marginal cost. ${ }^{7}$ Formally,

Definition 1 A contest is "easy" when $g$ ' is strictly concave $(f$ convex $)$. Conversely, a contest is "hard" when $g^{\prime}$ is strictly convex ( $f$ concave).

\footnotetext{
${ }^{6}$ A model with linear costs can be considered as a special case of the easy contests presented in this paper. As a result of the stark free-riding, it would be optimal to allocate the entire prize to the player with the lowest marginal cost.

${ }^{7}$ The form of the marginal cost, $g^{\prime}$, depends on its third derivative. There are contests that are neither "easy" nor "hard", for example when $g^{\prime \prime \prime}(x)>0$ for some $x$, and $g^{\prime \prime \prime}(x)<0$ for others. However, we focus our analysis only on these two cases.
} 


\section{A model of group contests with managers}

Hereafter, we move away from the model with complete information and exogenous predetermined prize allocations. Instead, we now assume that every group has a manager that sets a prize allocation $\phi_{i}=\left(\phi_{i 1}, \ldots, \phi_{i n_{i}}\right)$ s.t. $\sum_{k=1}^{n_{i}} \phi_{i k}=1$ in order to maximize her group's probability of winning. ${ }^{8}$ This is a common situation since the compensation of managers is usually aligned with the results of their group. In addition, for a matter of realism, we assume that the prize allocation implemented by each manager is unobservable by those belonging to other groups. However, all players' abilities remain common knowledge.

\subsection{Information Structure}

The timing and the information structure are adapted from Nitzan and Ueda (2011, 2018) and described as follows: (i) each manager announces the prize allocation $\phi_{i}=\left(\phi_{i 1}, \ldots, \phi_{i n_{i}}\right)$ to her members and (ii) group members enter in the contest without knowing the prize allocations implemented in the other groups, and determine their contributions simultaneously and noncooperatively. Before choosing his contribution, group member $i k$ only knows his own group prize allocation, and finds himself in the information set containing the nodes at which the other groups have chosen a prize allocation $\phi_{j \neq i}=\left(\phi_{j 1}, \ldots, \phi_{j n_{j}}\right)$. Thus, member $i k$ 's strategy is described as a function of $\phi_{i}$, and denoted by $x_{i k}\left(\phi_{i}\right)$.

\subsection{Beliefs}

We consider the perfect Bayesian equilibrium as a solution of our model assuming that players can use only pure strategies. Since the choice of the prize allocation $\phi_{i}=\left(\phi_{i 1}, \ldots, \phi_{i n_{i}}\right)$ is simultaneously made at the beginning of the game by the managers, then group members' beliefs are trivial. The belief of player $i k$, denoted by $\mu_{i k}\left(\phi_{i}\right)$, is a probability distribution defined over the space of possible allocations implemented in other groups. Suppose that $\left(\phi_{1}^{*}, \ldots, \phi_{N}^{*}\right)$ is an equilibrium prize allocation. At the information set lying on the equilibrium path the requirement of consistency implies that player's $i k$ belief satisfies $\mu_{i k}\left(\phi_{j \neq i}^{*} \mid \phi_{i}^{*}\right)=1$. Finally, we restrict the beliefs of group members off the path appealing to the "no-signalling-what-youdon't-know" condition. ${ }^{9}$ Thus, any deviation by a manager does not change the beliefs of her group members about the allocations implemented in other groups, i.e. $\mu_{i k}\left(\phi_{j \neq i}^{*} \mid \phi_{i}\right)=1 \forall \phi_{i}$. Altogether, we can use Eq. (2) to characterize player $i k$ and group $i$ 's best responses. Since members are aware of the prize allocation $\phi_{i}=\left(\phi_{i 1}, \ldots, \phi_{i n_{i}}\right)$ implemented by their own manager, i.e. at the information set indexed by $\phi_{i}$, then the best responses are

\footnotetext{
${ }^{8}$ We could relax the equality constraint to $\sum_{k=1}^{n_{i}} \phi_{i k} \leq 1$, but it would not change our results since we focus on the case in which managers aim to maximize their group's effort .

${ }^{9}$ Fudenberg and Tirole (1991).
} 


$$
X_{i}^{*}\left(\phi_{i}\right)=\sum_{k=1}^{n_{i}} x_{i k}^{*}\left(\phi_{i}\right)=\sum_{k=1}^{n_{i}} f\left(\frac{X_{j \neq i}^{*}\left(\phi_{j}^{*}\right)}{X^{2}} \phi_{i k} v_{i k}\right) .
$$

As previously established, players $i k$ 's expected payoff is strictly concave in $x_{i k}$. It follows that the first-order conditions given by Eq. (4) are necessary and sufficient for the best responses.

\subsection{Efficient managers}

Managers' behaviours vary accordingly to their objectives. We consider the case in which they want to maximize their group's effort through the prize allocation $\phi_{i}=\left(\phi_{i 1} \ldots, \phi_{i n_{i}}\right)$. Before moving forward, it is important to note that the members of a group hold identical beliefs about the prize allocations implemented in other groups. Thus, if a manager maximizes her own group effort $X_{i}\left(\phi_{i}\right)$, then she also maximizes her group probability of winning since $\sigma_{i}=X_{i}\left(\phi_{i}\right) /\left(X_{i}\left(\phi_{i}\right)+X_{j}^{*}\left(\phi_{j}^{*}\right)\right)$. This observation allows us to see that the maximization of the group effort $X_{i}\left(\phi_{i}\right)$ and the maximization of the group probability of winning $\sigma_{i}$ are two equivalent problems. Overall, the manager of group $i$ has to solve

$$
\phi_{i}^{*} \in \operatorname{argmax} X_{i}^{*}\left(\phi_{i}\right) \text { s.t. } \sum_{k=1}^{n_{i}} \phi_{i k}=1, \phi_{i k} \geq 0 \forall k,
$$

where $X_{i}^{*}\left(\phi_{i}\right)$ is defined by Eq. (4). Hence, if we find a profile of prize allocations $\left(\phi_{i}^{*}, \ldots, \phi_{N}^{*}\right)$ that solves (5) for all $i$, and all players maximize their expected payoff under their information set, i.e. Eq. (4) holds with equality for all $i$, then we can state that it is a pure-strategy perfect Bayesian equilibrium of the model with managers. As established by the following propositions, the number of equilibria depends on the type of contest and group members' ability.

Proposition 3.1 Given Assumption 1, the easy contest between groups with managers has $\prod_{i}^{N} n_{i}^{h}$ perfect Bayesian equilibria in pure strategies, where $n_{i}^{h}$ is the number of group members with the highest ability in group i. Moreover,

i) every $\phi_{i}^{*}$ rewards the entire prize to one of the $n_{i}^{h}$ group members;

ii) all equilibria provide the same $X_{i}^{*}, \sigma_{i}^{*}$, and $X^{*} \forall i$.

Since an equilibrium is a profile of prize allocations $\left(\phi_{i}^{*}, \ldots, \phi_{N}^{*}\right)$ that solves the systems of Eqs. (4) and (5), and $\phi_{i}^{*}$ rewards the entire prize to one among the most able group members, then all the optimal allocations provide the same $X_{i}^{*}$ for all $i$. However, at different equilibrium allocations different members contribute to the group effort. On the other hand, a unique equilibrium with one optimal prize allocation exists if the contest is hard. 
Proposition 3.2 Given Assumption 1, the hard contest between groups with managers has a unique perfect Bayesian equilibrium in pure strategies. Moreover,

i) symmetric players are rewarded equally, $v_{i k}=v_{i m}$ implies $\phi_{i k}^{*}=\phi_{i m}^{*}$;

ii) if marginal productivity is unbounded, then all members receive a positive share of the prize, $g^{\prime \prime}(0)=0$ implies $\phi_{i k}^{*}>0 \forall k$;

iii) if marginal productivity is bounded above, then only the highest ability members receive a positive share of the prize for any equilibrium effort, $g^{\prime \prime}(0)>0$ implies $\phi_{i k}^{*}>0 \forall X^{*}$ iff $v_{i k}=\max [v]$.

In hard contests, a prize allocation is optimal when all the members of a group have the same marginal productivity, i.e. if $\frac{v_{i k}}{g^{\prime \prime}\left(x_{i k}^{*}\right)}=\frac{v_{i m}}{g^{\prime \prime}\left(x_{i m}^{*}\right)}$ where $m \neq k .{ }^{10}$ Thus, group members with the same ability receive the same share of the prize $\phi_{i k}^{*}$. Furthermore, if $g^{\prime \prime}(0)=0$, then all players always receive a positive share of the prize since their marginal productivity at zero effort equals infinity. Conversely, if $g^{\prime \prime}(0)>0$, then the players' marginal productivity are bounded above. As a result, only the highest ability members of every group are certain of receiving a positive share of the prize. For example, we may have that $\frac{v_{i 1}}{g^{\prime \prime}\left(X_{i}^{*}\right)} \geq \frac{v_{i m}}{g^{\prime \prime}(0)}$, i.e. player $i 1$ receives the entire prize because we do not allow for negative prizes. We can relate our results to Cornes and Hartley (2005). In general terms, the authors define a player's dropout point as the equilibrium effort $X^{*}$ that makes him a non-active participant. If $g^{\prime}(0)=0$, then there is no dropout point and all players exert positive effort. As a matter of facts, in our setting, all groups are always active, but member im drops out if $v_{i m}<v_{i 1}$ in easy contests, and if $v_{i m} / g^{\prime \prime}(0) \leq v_{i 1} / g^{\prime \prime}\left(x_{i 1}^{*}\right) \forall m>1$ in hard ones. However, group members do not drop out from the competition voluntarily, but they optimally respond to the allocation implemented by their manager. Part (iii) of the proposition can be related to the group size effect in contests. Specifically, the literature shows that, in hard contests in which groups are formed by symmetric members who equally share the prize, a group increases its effort increasing its size. ${ }^{11}$ In contrast, in our setting that introduces heterogeneity, an increase in size affects the group effort if and only if the new member receives a positive share of the prize from the optimal prize allocation. Finally, the two propositions above relates to inequality if we interpret the optimal allocation as the efficient (reverse) Pigou-Dalton transfer of the (possible) rewards. ${ }^{12}$ Thus, when the contest is easy and/or players are heterogeneous, then managers have always a preference for inequality. Specifically, they would commit to transfer part of (or all) the possible gains from the less able workers to the most able ones even if the contest is hard.

\footnotetext{
10 Equivalently, for any two active members it holds $\frac{g^{\prime}\left(x_{i 1}^{*}\right)}{g^{\prime \prime}\left(x_{i 1}^{*}\right) \phi_{i 1}^{*}}=\frac{g^{\prime}\left(x_{i m}^{*}\right)}{g^{\prime \prime}\left(x_{i m}^{*}\right) \phi_{i m}^{*}} \forall m>1$.

11 See the "anti- Olson (1965) theorem" in Nitzan and Ueda (2014) and Proposition 2 in Esteban and Ray (2001).

12 I am grateful to an anonymous referee for bringing this point to my attention.
} 


\subsection{Within group heterogeneity and group effectiveness}

In this section, we revise some results of the effects of within group heterogeneity in ability under the widely used egalitarian allocation, ${ }^{13} \phi_{i k}=1 / n_{i} \forall k$, and then compare them to the effects of heterogeneity under the optimal allocation, $\phi_{i}=\phi_{i}^{*} \forall i$. To consider this matter, we define heterogeneity within groups following the definition of inequality in the sense of Lorenz dominance. Thus, we ask when $X_{i}^{\prime}\left(x_{i k}^{\prime}, \ldots, x_{i n_{i}}^{\prime}\right) \geq X_{i}\left(x_{i 1}, \ldots, x_{i n_{i}}\right)$, where $x_{i 1}^{\prime}, \ldots, x_{i n_{i}}^{\prime}$ are members equilibrium efforts under a "more spread out" distribution of abilities than $x_{i 1}, \ldots, x_{i n_{i}}$. To go along with this analysis we define the notions associated with majorization introduced by Hardy et al. (1934) which is equivalent to the notion of inequality in the sense of Lorenz dominance as shown by Dasgupta et al. (1973).

Definition 2 Let $\mathbf{x}^{\prime}$ and $\mathbf{x}$ be two vectors in $R^{n}$, ordered so that $x_{1}^{\prime} \geq \ldots \geq x_{n}^{\prime}$ and $x_{1} \geq \ldots \geq x_{n}$. If $\sum_{k=1}^{n} x_{k}^{\prime}=\sum_{k=1}^{n} x_{k}$ and $x_{1}^{\prime}+\ldots+x_{l}^{\prime} \geq x_{1}+\ldots+x_{l}$ for all $l \leq n$ (with strict inequality for at least one $l$ ), then we say that $\mathbf{x}^{\prime}$ majorizes $\mathbf{x}$ written as $\mathbf{x}^{\prime}>\mathbf{x}$. A permutation symmetric function $F$ of $n$ variables is Schur-convex if the inequality $F\left(\mathbf{x}^{\prime}\right) \geq F(\mathbf{x})$ holds whenever $\mathbf{x}^{\prime}>\mathbf{x}$. General discussion of majorization theory and Schur-convex functions can be found in Marshall et al. (1979).

Thus, we try to understand in which situations within group heterogeneity increases group effectiveness drawing on existing knowledge from non-strategic environments, but keeping in mind that we actually move towards different equilibria. Let us now assume that all managers implement the egalitarian allocation, $\phi_{i}=1 / n_{i} \forall i$. In this situation, the group $i$ 's effort in equilibrium is

$$
X_{i}^{*}=\sum_{k=1}^{n_{i}} f\left(\frac{1-\sigma_{i}^{*}}{X^{*}} \frac{1}{n_{i}} v_{i k}\right) .
$$

Fixing $\sigma_{i}^{*}$ and $X^{*}$, the group i's effort can be written as a function of the vector of abilities, $X_{i}^{*}=F\left(\mathbf{v}_{\mathbf{i}}\right)$. This observation together with Definition 2 helps us to state ${ }^{14}$

Lemma 2 Given a contest between groups in which the prize is equally shared among group members:

i) if the contest is easy, then the higher the within group heterogeneity in ability, the higher the group effectiveness. Formally, a change from $\mathbf{v}_{\mathbf{i}}$ to $\mathbf{v}_{\mathbf{i}}^{\prime}$ where $\mathbf{v}_{\mathbf{i}}^{\prime}>\mathbf{v}_{\mathbf{i}}$ implies $\sigma_{i}^{\prime}>\sigma_{i}, X^{\prime}>X$ and $X_{i}^{\prime}>X_{i}$.

\footnotetext{
${ }^{13}$ This is mathematically equivalent to a public good contest.

${ }^{14}$ Lemma 2 can be derived from Proposition 2 in Nitzan and Ueda (2014) where they allow heterogeneity in $\phi_{i k}$ fixing $v_{i k}$.
} 
ii) if the contest is hard, then the lower the heterogeneity in ability, the higher the group effectiveness. Formally, a change from $\mathbf{v}_{\mathbf{i}}$ to $\mathbf{v}_{\mathbf{i}}^{\prime}$ where $\mathbf{v}_{\mathbf{i}}^{\prime} \prec \mathbf{v}_{\mathbf{i}}$ implies $\sigma_{i}^{\prime}>\sigma_{i}$, $X^{\prime}>X$ and $X_{i}^{\prime}>X_{i}$

The intuition of this result is easy to grasp: when the contest is easy, low ability players free-ride on high ability ones who have a lower cost of contributing. On the other hand, high ability players are willing to exert substantial amounts of effort that more than compensate for the free-riding since their cost (for additional contributions) does not increase rapidly. Hence, keeping the average group ability constant, the greater the heterogeneity within a group, the higher its effectiveness, or, equivalently, a more spread out (unequal) distribution of abilities within a group increases its effectiveness. In hard contests, however, the cost of additional amounts of effort increases so rapidly that works as a deterrent for all players, but especially for the highly skilled that recede from exerting substantial contributions. So, when players are equally rewarded, a lower heterogeneity weakens this effect by making players exert similar (and less costly at the margin) efforts. Similarly, a less spread out distribution of abilities implies that members share the costs more equally, thus paying a lower cost per unit of group effort. A natural question to ask is whether the above result extends to our framework with optimal incentives where managers, assigning specific incentives, can enlarge the range of the possible contributions of their members, which now depends both on abilities and prize shares. The study of this matter under the implementation of the optimal allocation $\phi_{i}^{*}$ is straightforward for easy contests because the highest ability players always receive the entire prize. On the other hand, for hard contests, we have to carefully analyse players' and managers' behaviours. Indeed, any change in ability distribution leads to a change in the optimal allocation $\phi_{i}^{*}$, together with changes in the group effort $X_{i}^{*}$ and aggregate effort $X^{*}$. Thus, to make this analysis tractable we assume that $g^{\prime \prime}(0)=0$, which implies that all group members always receive a positive share of the prize, i.e. $\phi_{i k}^{*}>0 \forall v_{i k}>0 .{ }^{15}$ Altogether, the equilibrium group $i$ 's effort under the optimal prize allocation is

$$
X_{i}^{*}=\sum_{k=1}^{n_{i}} f\left(\frac{1-\sigma_{i}^{*}}{X^{*}} v_{i k} \phi_{i k}^{*}\right) .
$$

Since the prize allocation is a function of the distribution of abilities within the group, fixing $\sigma_{i}^{*}$ and $X^{*}$ allows us to write the group $i$ 's effort as a function of the vector of abilities, $X_{i}^{*}=F\left(\mathbf{v}_{\mathbf{i}}\right)$. This observation and Definition 2 help us to state the next proposition. ${ }^{16}$

\footnotetext{
15 The widely used power cost function $x^{\alpha} / b$ satisfies this condition.

16 Condition on part (ii-iii) is similar to measures of cautiousness, i.e. $\left(g^{\prime \prime \prime} / g^{\prime \prime}\right) /\left(g^{\prime \prime} / g^{\prime}\right)$. Similar versions of it appears prominently in the contest literature, for example see lemma 3 in Akerlof and Holden (2012) and Proposition 1 in Ryvkin (2011). However, $g(x)$ is a deterministic cost function, hence these notions are not directly relevant to the situation under study.
} 
Proposition 3.3 Given a contest between groups with managers:

i) if the contest is easy, then an increase in within group heterogeneity raises the group effectiveness. Formally, a change from $\mathbf{v}_{\mathbf{i}}$ to $\mathbf{v}_{\mathbf{i}}^{\prime}$ where $\mathbf{v}_{\mathbf{i}}^{\prime}>\mathbf{v}_{\mathbf{i}}$ implies $\sigma_{i}^{\prime} \geq \sigma_{i}$, $X^{\prime} \geq X$, and $X_{i}^{\prime} \geq X_{i}$

ii) if the contest is hard, $g^{\prime \prime}(0)=0$ and $g^{\prime \prime}(x) / g^{\prime}(x)>2 g^{\prime \prime \prime}(x) / g^{\prime \prime}(x)$, an increase in within group heterogeneity raises the group effectiveness. Formally, a change in the distribution of ability from $\mathbf{v}_{\mathbf{i}}$ to $\mathbf{v}_{\mathbf{i}}^{\prime}$ where $\mathbf{v}_{\mathbf{i}}^{\prime}>\mathbf{v}_{\mathbf{i}}$ implies $\sigma_{i}^{\prime}>\sigma_{i}, X^{\prime}>X$, and $X_{i}^{\prime}>X_{i}$

iii) if the contest is hard, $g^{\prime \prime}(0)=0$ and $g^{\prime \prime}(x) / g^{\prime}(x)<2 g^{\prime \prime \prime}(x) / g^{\prime \prime}(x)$, then a decrease in within group heterogeneity raises the group effectiveness. Formally, a change in the distribution of ability from $\mathbf{v}_{\mathbf{i}}$ to $\mathbf{v}_{\mathbf{i}}^{\prime}$ where $\mathbf{v}_{\mathbf{i}}^{\prime} \prec \mathbf{v}_{\mathbf{i}}$ implies $\sigma_{i}^{\prime}>\sigma_{i}, X^{\prime}>X$, and $X_{i}^{\prime}>X_{i}$;

Common to the literature of contests is the assumption that either groups implement the egalitarian allocation or group members are symmetric in their ability. Thus, the analysis of within group heterogeneity follows directly from Lemma 2. As a matter of fact, it is widely argued in the literature that in hard contests groups are more effective the less their within group heterogeneity. Examples include Nitzan and Ueda (2014), Cubel and Sanchez-Pages (2014), and Esteban and Ray (2001). However, as shown by part (ii), these results do not hold under the implementation of the optimal rule. For example, let the cost function be $x^{\alpha}$, and the related marginal costs $g^{\prime}(x)=\alpha x^{(\alpha-1)}$. It is easy to see that a contest is hard for any $\alpha>2$, and $g^{\prime \prime}(x) / g^{\prime}(x)>2 g^{\prime \prime \prime}(x) / g^{\prime \prime}(x) \forall \alpha \in(2,3)$, which implies that for $2<\alpha<3$ within group heterogeneity increases group efficiency under the use of the optimal allocation. Thus, the deterrent effect that induced highly skilled players to recede from exerting substantial contributions can be balanced out by assigning them higher shares of the prize. ${ }^{17}$ Indeed, if the initial share is the same, then an increment of the allocated prize induces more effort from the more able individual. Altogether, a more spread out distribution of abilities is efficient if the complementarity between ability and rewards boosts enough skilled players efforts to more than compensate for the deterrent effect of the increase in costs. ${ }^{18}$ We conclude our analysis highlighting other relevant results related to the use of the optimal prize allocation. As shown in the following propositions, which follow directly from Proposition 3.3, we can rank groups' probability of winning. Such a ranking is not possible in easy contests

17 If $g^{\prime \prime}(x) / g^{\prime}(x)>2 g^{\prime \prime \prime}(x) / g^{\prime \prime}(x)$, then $g^{\prime}(x) / g^{\prime \prime}(x)$ is increasing (see Eq. (23)), which implies that higher ability players receive higher share of the prize. Interestingly, this is not generally true.

${ }^{18}$ The same intuition can be explained looking at the marginal costs of contributing per unit of group effort via a simple example: a highly skilled player with ability $V$ and a group of $n$ symmetric players with ability $V / n$ are competing for a prize $P$. The marginal cost of group effort are $\frac{X^{\alpha-1}}{V}$ and $\left(\frac{X}{n}\right)^{\alpha-1} \frac{n^{2}}{V}$ respectively. Finally, it is easy to see that if $1<\alpha<3$ the single player has lower costs and then he exerts higher effort in equilibrium. 
with heterogeneous groups under the implementation of other incentive mechanisms such as the relative effort rule and the egalitarian rule.

Proposition 3.4 Consider a contest with $N$ groups formed by $n_{i}$ individuals such that $\mathbf{v}_{\mathbf{1}}>\ldots>\mathbf{v}_{\mathbf{N}}$. If the contest is easy and $\phi_{i}=\phi_{i}^{*} \forall i$, then groups' probability of winning can be ordered according to the highest ability members in every group. Formally, $v_{i 1}>\ldots>v_{N 1}$ implies $\sigma_{1}>\ldots>\sigma_{N}$;

Thus, if managers optimally allocate the prize, differences in sizes between groups are irrelevant to the group efficiency. Indeed, groups can be ranked according to the most skilled member in every group. In addition to this, for some specific hard contests, the ranking resulting from the egalitarian allocation can be fully reversed by implementing the optimal prize allocation.

Proposition 3.5 Consider a hard contest with $N$ groups formed by $n_{i}=n$ individuals such that $\mathbf{v}_{\mathbf{1}}>\ldots>\mathbf{v}_{\mathbf{N}}$. Let $g^{\prime \prime}(0)=0$, and $g^{\prime \prime}(x) / g^{\prime}(x)>2 g^{\prime \prime \prime}(x) / g^{\prime \prime}(x)$, then the use of the egalitarian allocation $\phi_{i}=1 / n$ implies $\sigma_{1}<\ldots<\sigma_{N}$, while the optimal allocation $\phi_{i}=\phi_{i}^{*}$ implies $\sigma_{1}>\ldots>\sigma_{N}$.

\subsection{A full example with power cost functions}

Let $g(x)=x^{\alpha}$, which for $\alpha>1$ satisfies Assumption 1. Group i's best response, when members are aware of the prize allocation $\phi_{i}=\left(\phi_{i 1}, \ldots, \phi_{i n_{i}}\right)$ implemented by their own manager, is given by

$$
X_{i}^{*}\left(\phi_{i}\right)=\sum_{k=1}^{n_{i}} x_{i k}^{*}\left(\phi_{i}\right)=\sum_{k=1}^{n_{i}}\left(\frac{X_{j \neq i}^{*}\left(\phi_{j}^{*}\right)}{X^{2}} \phi_{i k} v_{i k}\right)^{\frac{1}{\alpha-1}}
$$

In order to maximize their group's effort managers have to solve

$$
\phi_{i}^{*} \in \operatorname{argmax} X_{i}^{*}\left(\phi_{i}\right) \text { s.t. } \sum_{k=1}^{n_{i}} \phi_{i k}=1, \phi_{i k} \geq 0 \forall k .
$$

From Proposition 3.2 we know that $\phi_{i k}^{*}>0 \forall k$ if $\alpha \in(2, \infty)$ (hard contest), while $\phi_{i 1}=1$ if $\alpha \in(1,2)$ (easy contest). Moreover, having assumed a specific cost function, the solution of (9) is

$$
\phi_{i k}^{*}=\frac{v_{i k}^{\frac{1}{\alpha-2}}}{\sum_{k=1}^{n_{i}} v_{i k}^{\frac{1}{\alpha-2}}} .
$$

Finally, substituting (10) into (8) and rearranging, gives us the group $i$ efforts in equilibrium of our model of contests with managers. 


$$
X_{i}= \begin{cases}\left(\frac{1-\sigma_{i}^{*}}{\alpha X^{*}} v_{i 1}\right)^{\frac{1}{\alpha-1}} & \text { if } \alpha \in(1,2] \\ \left(\frac{1-\sigma_{i}^{*}}{\alpha X^{*}}\right)^{\frac{1}{\alpha-1}}\left(\sum_{k=1}^{n_{i}} v_{i k}^{\frac{1}{\alpha-2}}\right)^{\frac{\alpha-2}{\alpha-1}} & \text { if } \alpha \in(2, \infty)\end{cases}
$$

Proposition 3.6 Given a contest between groups in which the prize is optimally allocated among group members:

i) if $1<\alpha<3$, then the higher the heterogeneity in ability the higher the group effort.

ii) if $\alpha>3$, then the lower the heterogeneity in ability the higher the group effort.

ii) If $\alpha \rightarrow \infty$, then $\phi_{i k} \rightarrow 1 / n_{i} \forall i, k$.

The above proposition shows that the category of contests for which heterogeneity increases group effectiveness is larger under the implementation of the optimal prize allocation than the egalitarian one. Specifically, it moves from $1<\alpha<2$, for the egalitarian allocation, to $1<\alpha<3$, for the optimal one. Finally, we can establish from Eq. (10) that the share of the prize that players receive depends on parameter $\alpha$ as follows: the higher the $\alpha$ the more equal the prize division among group members. It follows that, when the contest gets extremely hard, the optimal allocation tends to the egalitarian rule.

\section{Conclusions}

We have examined a model of group contests for a private good, in which individual contributions are not observable, to provide a prize allocation that maximizes groups' effectiveness. Our main findings are the following: in easy contests it is optimal to allocate the entire prize to one of the most able group members; in hard contests the optimal allocation depends on players' ability and their marginal productivity of effort; we provide sufficient conditions that make, in contrast with other results in the literature, heterogeneous groups more effective than homogeneous groups even in contests with strictly convex marginal costs.

Our model is general in the sense that it can be applied to many types of conflicts and work environments which encourage competition through specific incentive schemes. Moreover, it does not require that managers observe the contributions of every group member, a requirement that is necessary to implement the relative effort rule. Thus, we can advise managers on how to assign incentives and build their teams in different situations. For instance, in competitions with symbolic rewards, such as "best store of the month", we can advise managers to form a heterogeneous group to prevent free-riding problems when the cost function is not too steep. On the other hand, in retail firms that set up monetary 
reward contests for sales departments during periods with a positive shock or a peak in the demand for goods, such as the run-up to Christmas, we can suggest to the team managers to divide the prize among all group members to increase the team productivity assuming that the extra work provided by the workers substantially increases their marginal costs. In addition, our analysis on within group heterogeneity reveals new insights on inequality in conflicts. Specifically, it shows that a more spread out distribution of ability increases group effectiveness for hard contests under the condition that groups implement the optimal prize allocation. The simplicity of our framework is attractive but might be criticized because the group managers do not exert effort and have one goal: to maximize their own team's effectiveness. We could have assumed that team managers may contribute to their group's effort, but it would not have changed our intuitions based on the contest's categorization. Moreover, it is implicit in the model that managers maximize group efficiency because this gives them some direct or indirect benefits aligned with their teams' results; for example, job promotions or other monetary awards.

Open Access This article is licensed under a Creative Commons Attribution 4.0 International License, which permits use, sharing, adaptation, distribution and reproduction in any medium or format, as long as you give appropriate credit to the original author(s) and the source, provide a link to the Creative Commons licence, and indicate if changes were made. The images or other third party material in this article are included in the article's Creative Commons licence, unless indicated otherwise in a credit line to the material. If material is not included in the article's Creative Commons licence and your intended use is not permitted by statutory regulation or exceeds the permitted use, you will need to obtain permission directly from the copyright holder. To view a copy of this licence, visit http://creativecommons.org/licen ses/by/4.0/.

\section{Appendix A}

Proof of Lemma 1 The following proof is an extension of Ryvkin (2011) for a perfectly divisible prize. Player $i k$ 's best response function has to satisfy

$$
\frac{X_{j \neq i}}{X^{2}}=\left(v_{i k} \phi_{i k}\right)^{-1} g^{\prime}\left(x_{i k}\right) \text {. }
$$

Note that the left-hand side of Eq. (12) is the same for any player $k$ of group $i$. Without loss of generality, let $\phi_{i 1}>0$. It follows that for any $x_{i 1}$ we have $\left(v_{i m} \phi_{i m}\right)^{-1} g^{\prime}\left(x_{i m}\right)=\left(v_{i 1} \phi_{i 1}\right)^{-1} g^{\prime}\left(x_{i 1}\right)$. Thus, the effort exerted by all $i m$, where $m>1$, can be uniquely determined as a share of the effort exerted by player $i 1$ as

$$
x_{i m}=g^{(-1)}\left(\frac{v_{i m} \phi_{i m}}{v_{i 1} \phi_{i 1}} g^{\prime}\left(x_{i 1}\right)\right) .
$$

The group $i$ 's effort $X_{i}$ can be written as 


$$
\alpha_{i}\left(x_{i 1}\right)=x_{i 1}+\sum_{m>1}^{n_{i}} g^{\prime(-1)}\left(\frac{v_{i m} \phi_{i m}}{v_{i 1} \phi_{i 1}} g^{\prime}\left(x_{i 1}\right)\right),
$$

and using (13), the related marginal cost as

$$
G_{i}^{\prime}\left(x_{i 1}\right)=\sum_{k}^{n_{i}} \frac{g^{\prime}\left(x_{i k}\right)}{v_{i k}} .
$$

Functions $\alpha_{i}\left(x_{i 1}\right)$ and $G_{i}^{\prime}\left(x_{i 1}\right)$ are strictly increasing and satisfies $\alpha_{i}(0)=0$ and $G_{i}^{\prime}(0)=0$. Therefore, the contests among groups reduces to a contest among $N$ individuals:

$$
\frac{1-\sigma_{i}}{\sum_{i=1}^{N} \alpha_{i}\left(x_{i 1}\right)}=G^{\prime}\left(x_{i 1}\right) .
$$

Let $X_{i}=\alpha_{i}\left(x_{i 1}\right), x_{i 1}=\alpha^{-1}\left(X_{i}\right)$. Define $G_{i}\left(X_{i}\right)=\int_{0}^{X_{i}} G^{\prime}\left(\alpha^{-1}(t)\right) d t$ with initial conditions $G_{i}\left(X_{i}\right)=0 . G_{i}\left(X_{i}\right)$ is strictly increasing, strictly convex and satisfies Assumption 1 . The group $i$ 's expected payoff can be written as

$$
\frac{X_{i}}{X}-G_{i}\left(X_{i}\right)
$$

The uniqueness of equilibrium follows from Theorem 3 of Cornes and Hartley (2005).

Proof of Proposition 3.1 Let $g^{\prime}(x)$ be strictly concave and $f(x)$ strictly convex (easy contest). A profile of allocations $\left(\phi_{1}^{*}, \ldots, \phi_{N}^{*}\right)$ is a perfect Bayesian equilibrium if it satisfies Eqs. (4) and (5) $\forall i$.

In order to prove the proposition we use the following observations:

i) Equation (4) holds with equality for any prize allocation, i.e. for any $\phi_{i}=\left(\phi_{i 1}, \ldots, \phi_{i n_{i}}\right)$ there exists only one $X_{i}$ (the opposite is not true);

ii) Equation (4) is a sum of strictly convex functions for any fixed $X=X_{i}+X_{j \neq i}^{*}\left(\phi_{j}^{*}\right)>0 .{ }^{19}$ Hence, it is strictly convex.

Let us define the group $i$ 's effort that satisfies Eq. (4) for a prize allocation $\phi_{i}^{\prime}$ as $X_{i}\left(\phi_{i}^{\prime}, X_{j}^{*}\left(\phi_{j}^{*}\right)\right)=X_{i}^{\prime}($ see observation $\mathrm{i})$. Then, we can find an alternative allocation, $\phi_{i}^{a}$, which provides higher effort than $\phi_{i}^{\prime}$, as follows: we fix the total effort at $X=X_{i}^{\prime}+X_{j}^{*}\left(\phi_{j}^{*}\right)$; and we maximize the group $i$ 's effort given by Eq. (4). Clearly, the solution of this maximization problem lies in a corner (see observation ii). There are

19 Assumption 1 guarantees that if $\phi_{i k}>0$ for at least a $k$ in every group, then $X_{i}>0 \forall i$. 
$n_{i}^{h}$ allocations, where $n_{i}^{h}$ is the number of players with the highest ability in group $i$. Under the allocation $\phi_{i}^{a}$ we have that

$$
X_{i}^{\prime}<\sum_{k=1}^{n_{i}} f\left(\frac{X_{j}^{*}\left(\phi_{j}^{*}\right)}{\left(X_{i}^{\prime}+X_{j}^{*}\left(\phi_{j}^{*}\right)\right)^{2}} v_{i k} \phi_{i k}^{a}\right) .
$$

The first order condition is satisfied for $X_{i}^{a}>X_{i}^{\prime}$ such that $X_{i}^{a}=\sum_{k=1}^{n_{i}} f\left(\frac{X_{j}^{*}\left(\phi_{j}^{*}\right)}{\left(X_{i}^{a}+X_{j}^{*}\left(\phi_{j}^{*}\right)\right)^{2}} v_{i k} \phi_{i}^{a}\right)$. It is straightforward that every manager maximizes her group effort iff $\phi_{i}=\phi_{i}^{*}$ for any $X_{j}^{*}\left(\phi_{j}^{*}\right)>0$, i.e. she allocates the entire prize to one of the most able members. The uniqueness of equilibrium follows from Lemma 1.

Proof of Proposition 3.2 Let $g^{\prime}(x)$ be strictly convex and $f(x)$ strictly concave (hard contest).

In order to prove the proposition we use the observation (i) and (ii) stated for the proof of Proposition 3.1. First, we define the group $i$ 's effort that satisfies Eq. (4) for a particular prize allocation $\phi_{i}^{\prime}$ as $X_{i}\left(\phi_{i}^{\prime}, X_{j}^{*}\left(\phi_{j}^{*}\right)\right)=X_{i}^{\prime}$ using observation (i). Second, using observation (ii), we find an alternative allocation, $\phi_{i}^{a}$, which provides higher effort than $\phi_{i}^{\prime}$ as follows: we fix the total effort at $X=X_{i}^{\prime}+X_{j}^{*}\left(\phi_{j}^{*}\right)$; and we maximize the group $i$ 's effort given by Eq. (4). The solution of this maximization problem is interior, unique and implies

$$
X_{i}^{\prime}<\sum_{k=1}^{n_{i}} f\left(\frac{X_{j}^{*}\left(\phi_{j}^{*}\right)}{\left(X_{i}^{\prime}+X_{j}^{*}\left(\phi_{j}^{*}\right)\right)^{2}} v_{i k} \phi_{i k}^{a}\right) .
$$

The first order condition is satisfied for $X_{i}^{a}>X_{i}^{\prime}$ such that $X_{i}^{a}=\sum_{k=1}^{n_{i}} f\left(\frac{X_{j}^{*}\left(\phi_{j}^{*}\right)}{\left(X_{i}^{a}+X_{j}^{*}\left(\phi_{j}^{*}\right)\right)^{2}} v_{i k} \phi_{i}^{a}\right)$. Overall, we have to find the allocation that maximizes (5) at $X=X_{i}\left(\phi_{i}^{*}\right)+X_{j}^{*}\left(\phi_{j}^{*}\right)$, and the $X_{i}\left(\phi_{i}^{*}\right)$ that satisfies Eq. (4). The solution of Eq. (5) is given by

$$
\frac{\frac{1-\sigma_{i}}{X} v_{i k}}{g^{\prime \prime}\left(x_{i k}\right)}-\lambda=0 \quad \forall k .
$$

Let, without loss of generality, $v_{i 1} \geq \ldots \geq v_{i n_{i}}$. From the system of Eq. (16) we have that

$$
\frac{v_{i 1}}{g^{\prime \prime}\left(x_{i 1}\right)} \geq \frac{v_{i m}}{g^{\prime \prime}\left(x_{i m}\right)} \quad \forall m>1 .
$$

Using (17), the group $i$ 's effort $X_{i}\left(\phi_{i}^{*}\right)$ can be written as

$$
v_{i}\left(x_{i 1}\right)=x_{i 1}+\sum_{m>1}^{n_{i}} \max \left[0, g^{\prime \prime-1}\left(\frac{v_{i m}}{v_{i 1}} g^{\prime \prime}\left(x_{i 1}\right)\right)\right],
$$


and the related marginal costs as

$$
G_{i}^{\prime}\left(x_{i 1}\right)=\sum_{i k}^{n_{i}} \frac{g^{\prime}\left(x_{i k}\right)}{v_{i k}} .
$$

Functions $v\left(x_{i 1}\right)$ and $G^{\prime}\left(x_{i 1}\right)$ are strictly increasing and satisfy $v(0)=0, G^{\prime}(0)=0$. Therefore, the group contests reduces to a contests among $N$ individuals:

$$
\frac{1-\sigma_{i}}{\sum_{i=1}^{N} v_{i}\left(x_{i 1}\right)}=G_{i}^{\prime}\left(x_{i 1}\right) .
$$

Finally, let $X_{i}=v_{i}\left(x_{i 1}\right), x_{i 1}=v_{i}^{-1}\left(X_{i}\right)$. Define $G_{i}\left(X_{i}\right)=\int_{0}^{X_{i}} G^{\prime}\left(v_{i}^{-1}(t)\right) d t$ with initial conditions $G_{i}\left(X_{i}\right)=0 . G_{i}\left(X_{i}\right)$ is strictly increasing, strictly convex and satisfies Assumption 1. Group $i$ 's expected payoff can be written as

$$
\frac{X_{i}}{X}-G_{i}\left(X_{i}\right)
$$

The uniqueness of equilibrium follows from Theorem 3 of Cornes and Hartley (2005). The equilibrium effort $X_{i}^{*}$ implicitly defines the equilibrium allocation $\left(\phi_{1}^{*}, \ldots, \phi_{N_{i}}^{*}\right)$ through the relation $X_{i}^{*}=v_{i}\left(x_{i 1}^{*}\right)$. To better see that this solution gives a unique prize allocation for all $i$ consider the following contradiction. Suppose that the equilibrium effort $X_{i}^{*}$ can be obtained by two prize allocations, $\phi_{i}^{1}$ and $\phi_{i}^{2}$. This would imply that setting $X^{*}=X_{i}^{*}+X_{j}^{*}\left(\phi_{j}^{*}\right)$, and solving for Eq. (5) gives the two solutions $\phi_{i}^{1}$ and $\phi_{i}^{2}$. However, due to the strictly concavity of the best response function for fixed $X$ the solution is interior and unique.

Part i)

Recall that $X_{i}^{*}\left(\phi_{i}\right)>0$. Then, it is straightforward to see that the system of Eq. (17) holds with equality for all group members with the same ability $v_{i k}$ iff they receive the same prize share $\phi_{i k}^{*}$. Note that this does not imply that these members receive a positive share of the prize.

Part ii)

Let $g^{\prime \prime}(0)=0$, then $\frac{v_{i k}}{g^{\prime \prime}(0)}=\infty \forall k$. The optimal prize allocation satisfies the system of Eq. (17) with equality, i.e.

$$
\frac{v_{i 1}}{g^{\prime \prime}\left(x_{i 1}\right)}=\frac{v_{i m}}{g^{\prime \prime}\left(x_{i m}\right)} \quad \forall m>1 .
$$

It follows that all $i k$ receive a positive share of the prize $\phi_{i k}>0$. In addition, we can rewrite (16) as

$$
\frac{g^{\prime}\left(x_{i k}\right)}{g^{\prime \prime}\left(x_{i k}\right) \phi_{i k}}=\lambda \quad \forall k .
$$

Finally, using $\sum_{k}^{n_{i}} \phi_{i k}=1$, we can define the optimal prize allocation as 


$$
\phi_{i k}=\frac{\frac{g^{\prime}\left(x_{i k}\right)}{g^{\prime \prime}\left(x_{i k}\right)}}{\sum_{i k}^{n_{i}} \frac{g^{\prime}\left(x_{i k}\right)}{g^{\prime \prime}\left(x_{i k}\right)}} \forall k .
$$

\section{Part iii)}

Let $g^{\prime \prime}(0)>0$. The prize allocation of all players can be derived from the relation $\sigma_{i}^{*} X^{*}=X_{i}^{*}=v_{i}\left(x_{i 1}\right)=x_{i 1}+\sum_{m>1}^{n_{i}} \max \left[0, g^{\prime \prime-1}\left(\frac{v_{i m}}{v_{i 1}} g^{\prime \prime}\left(x_{i 1}\right)\right)\right]$. Moreover, if

$$
\frac{v_{i 1}}{g^{\prime \prime}\left(X_{i}^{*}\right)} \geq \frac{v_{i m}}{g^{\prime \prime}(0)} \quad \forall m>1
$$

then it holds

$$
\frac{v_{i 1}}{g^{\prime \prime}\left(f\left(\frac{1-\sigma_{i}^{*}}{X^{*}} v_{i 1}\right)\right)} \geq \frac{v_{i m}}{g^{\prime \prime}(0)} \quad \forall m>1
$$

Proof of Lemma 2 Given a distribution of ability $\mathbf{v}_{\mathbf{i}}=\left(v_{i 1}, \ldots, v_{i n}\right)$, the group $i$ 's total effort given by (6) can be rewritten as

$$
\sigma_{i}=\frac{\sum_{k=1}^{n_{i}} f\left(\frac{1-\sigma_{i}}{X} \frac{1}{n_{i}} v_{i k}\right)}{X} .
$$

Equation (24) allows us to see that for each given $X$ and $\mathbf{v}_{\mathbf{i}}$ there is a unique value of $\sigma_{i}$ that satisfies Eq. (6). In other words, Eq. (6) implicitly defines $\sigma_{i}$ as a function of $\mathbf{v}_{\mathbf{i}}$ and $X ; \sigma_{i}=\sigma_{i}\left(\mathbf{v}_{\mathbf{i}}, X\right)$. The equilibrium value of $X$ is then determined by the condition $\sum_{i=1}^{N} \sigma_{i}=1$. Moreover, $\sigma_{i}\left(\mathbf{v}_{\mathbf{i}}, X\right)$ is strictly decreasing and continuous in $X, \lim _{X \rightarrow \infty} \sigma_{i}=0$ and $\lim _{X \rightarrow 0} \sigma_{i}=1$. These properties of the share functions follow directly from Theorem 3 of Cornes and Hartley (2005). Now, we are ready to prove the Lemma examining the behaviour of $\sigma_{i}\left(\mathbf{v}_{\mathbf{i}}, X\right)$ moving from $\mathbf{v}_{\mathbf{i}}$ to $\mathbf{v}_{\mathbf{i}}^{\prime}$ when $f$ is strictly convex.

Let $\sigma_{i}^{*}$ and $X^{*}$ be equilibrium values under the ability vector $\mathbf{v}_{\mathbf{i}}$, i.e. $\sum_{i}^{N} \sigma_{i}^{*}\left(\mathbf{v}_{\mathbf{i}}, X^{*}\right)=1$, then:

i) fix $X^{*}$ and $\sigma_{i}^{*}$, the right hand side of (24) defined by $F\left(\mathbf{v}_{\mathbf{i}}\right)$ is Schur-convex. Using Definition 2, a change in ability distribution such that $\mathbf{v}_{\mathbf{i}}^{\prime}>\mathbf{v}_{\mathbf{i}}$ implies $F\left(\mathbf{v}_{\mathbf{i}}{ }^{\prime}\right)>F\left(\mathbf{v}_{\mathbf{i}}\right)$. The $\sigma_{i}$ that solves (24) for the new distribution of ability, but keeping $X^{*}$ fixed, is $\sigma_{i}^{\prime}\left(\mathbf{v}_{\mathbf{i}}{ }^{\prime}, X^{*}\right)>\sigma_{i}^{*}\left(\mathbf{v}_{\mathbf{i}}, X^{*}\right)$. However, this is not the new equilibrium since $\sigma_{i}^{\prime}\left(\mathbf{v}_{\mathbf{i}}{ }^{\prime}, X^{*}\right)+\sum_{j \neq i}^{N} \sigma_{j}\left(\mathbf{v}_{\mathbf{j}}, X^{*}\right)>1$

ii) the new equilibrium total effort $X^{* *}$ satisfies $\sigma_{i}\left(\mathbf{v}_{\mathbf{i}}{ }^{\prime}, X^{* *}\right)+\sum_{j \neq i}^{N} \sigma_{j}\left(\mathbf{v}_{\mathbf{j}}, X^{* *}\right)=1$. Hence, $X^{* *}>X^{*}$ since $\sigma_{i}\left(\mathbf{v}_{\mathbf{i}}, X\right)$ is continuous and strictly decreasing in $X \forall i$. This proves that for every group other than $i$ the winning probability strictly falls, i.e. $\sigma_{j}^{* *}\left(\mathbf{v}_{\mathbf{j}}, X^{* *}\right)<\sigma_{j}^{*}\left(\mathbf{v}_{\mathbf{j}}, X^{*}\right) \forall j \neq i$ and $\sigma_{i}^{* *}\left(\mathbf{v}_{\mathbf{i}}{ }^{\prime}, X^{* *}\right)>\sigma_{i}^{*}\left(\mathbf{v}_{\mathbf{i}}, X^{*}\right)$;

iii) finally, $\sigma_{i}^{* *}>\sigma_{i}^{*}$ and $X^{* *}>X^{*}$ imply $X_{i}^{* *}>X_{i}^{*}$.

The same analysis holds if $\mathbf{v}_{\mathbf{i}}^{\prime} \prec \mathbf{v}_{\mathbf{i}}$ and $f$ is strictly concave. 


\section{Proof of Proposition 3.3 Part i)}

Let $v_{i 1}$ be the highest ability in group $i$. In easy contests ( $f$ is convex) the optimal allocation $\phi_{i}=\phi_{i}^{*}$ rewards players as follows: $\phi_{i 1}=1, \phi_{i m}=0 \forall m>1$. It follows from Lemma 2 that a change in ability distribution such that $\mathbf{v}_{\mathbf{i}}^{\prime}>\mathbf{v}_{\mathbf{i}}$ and $v_{i 1}^{\prime}>v_{i 1} \rightarrow \sigma_{i}^{* *}>\sigma_{i}^{*}$. On the other hand, a change in ability distribution such that $\mathbf{v}_{\mathbf{i}}^{\prime}>\mathbf{v}_{\mathbf{i}}$ but $v_{i 1}^{\prime}=v_{i 1} \rightarrow \sigma_{i}^{* *}=\sigma_{i}^{*}$.

\section{Part ii-iii)}

In order to prove Part ii (iii) of the proposition, it is sufficient to show that at a fixed $X$ and $\sigma_{i}$ the right-hand side of (7) is Schur-Convex (Schur-Concave). The rest of the proof follows from Lemma 2.

Let $v_{i 1} \geq \ldots \geq v_{i n_{i}}$ and from (7) $F\left(\mathbf{v}_{\mathbf{i}}\right)=\sum_{k=1}^{n_{i}} f\left(\frac{1-\sigma_{i}^{*}}{X^{*}} v_{i k} \phi_{i k}^{*}\left(v_{i k}, \mathbf{v}_{\mathbf{i}}\right)\right) / X^{*}$. Fixing $X^{*}$ and $\sigma_{i}^{*}$, then $F\left(\mathbf{v}_{\mathbf{i}}\right)$ is Schur-Convex if it holds the Schur-Ostrowski criterion, $\frac{\partial F\left(\mathbf{v}_{\mathbf{i}}\right)}{\partial v_{i 1}}-\frac{\partial F\left(\mathbf{v}_{\mathbf{i}}\right)}{\partial v_{i m}}>0 \forall m>1$. Hence, if

$$
\frac{\phi_{i 1}^{*}}{g^{\prime \prime}\left(x_{i 1}\right)}-\frac{\phi_{i m}^{*}}{g^{\prime \prime}\left(x_{i m}\right)}+\sum_{i k}^{n_{i}} \frac{v_{i k} \frac{\partial \phi_{i k}^{*}}{\partial v_{i 1}}}{g^{\prime \prime}\left(x_{i k}\right)}-\sum_{i k}^{n_{i}} \frac{v_{i k} \frac{\partial \phi_{i k}^{*}}{\partial v_{i m}}}{g^{\prime \prime}\left(x_{i k}\right)}>0 .
$$

Since $g^{\prime \prime}(0)=0$ implies $\frac{v_{i 1}}{g^{\prime \prime}\left(x_{i 1}\right)}=\frac{v_{i m}}{g^{\prime \prime}\left(x_{i m}\right)}$ (see the proof of Proposition 3.2 part ii) and $\sum_{i k}^{n_{i}} \frac{\partial \phi_{i k}}{\partial v i m}=0$, then $\sum_{i k}^{n_{i}} \frac{v_{i k} \frac{\partial \phi_{i k}^{*}}{\partial v_{i m}}}{g^{\prime \prime}\left(x_{i k}\right)}=0$. Overall, $F\left(\mathbf{v}_{\mathbf{i}}\right)$ is Schur-Convex if

$$
\frac{\phi_{i 1}^{*}}{g^{\prime \prime}\left(x_{i 1}\right)}>\frac{\phi_{i m}^{*}}{g^{\prime \prime}\left(x_{i m}\right)}
$$

Substituting $\phi_{i k}^{*}$ with Eq. (23), we get

$$
\frac{g^{\prime}\left(x_{i 1}\right)}{g^{\prime \prime}\left(x_{i 1}\right)^{2}}>\frac{g^{\prime}\left(x_{i m}\right)}{g^{\prime \prime}\left(x_{i m}\right)^{2}}
$$

which holds when $g^{\prime \prime}(x) / g^{\prime}(x)>2 g^{\prime \prime \prime}(x) / g^{\prime \prime}(x)$; thereby $\quad \mathbf{v}_{\mathbf{i}}^{\prime}>\mathbf{v}_{\mathbf{i}}$ implies $F\left(\mathbf{v}_{\mathbf{i}}^{\prime}\right)>F\left(\mathbf{v}_{\mathbf{i}}\right)$ and $\sigma_{i}^{\prime}\left(\mathbf{v}_{\mathbf{i}}{ }^{\prime}, X^{*}\right)+\sum_{j>1}^{N} \sigma_{j}\left(\mathbf{v}_{\mathbf{j}}, X^{*}\right)>1$. Finally, part (ii-iii) of Lemma 2 concludes the proof.

Proof of Proposition 3.6 In order to maximize Eq. (8) with respect to $\phi_{i k}$, we have to solve the following Lagrangean problem:

$$
L=\sum_{k=1}^{n_{i}}\left(\frac{1-\sigma_{i}^{*}}{X^{*}}\left(v_{i k} \phi_{i k}\right)\right)^{\frac{1}{\alpha-1}}+\lambda\left(1-\sum_{k=1}^{n_{i}} \phi_{i k}\right),
$$

which gives

$$
f_{i 1}^{\prime}(.) v_{i 1}=f_{i m}^{\prime}(.) v_{i m}
$$

We can rewrite the share of the prize for any player $i m \neq i 1$ as a share of the prize received by player $i 1$ as 


$$
\phi_{i m}=\phi_{i 1}\left(\frac{v_{i m}}{v_{i 1}}\right)^{\frac{1}{\alpha-2}},
$$

and substituting it in the constraint gives

$$
\phi_{i 1}+\sum_{m=2}^{n_{i}} \phi_{i 1}\left(\frac{v_{i m}}{v_{i 1}}\right)^{\frac{1}{\alpha-2}}=1 .
$$

A simple rearrangement defines the optimal prize allocation as

$$
\phi_{i 1}=\frac{v_{i 1}^{\frac{1}{\alpha-2}}}{\sum_{k=1}^{n_{i}} v_{i k}^{\frac{1}{\alpha-2}}},
$$

and for every im as

$$
\phi_{i m}^{*}=\frac{v_{i m}^{\frac{1}{\alpha-2}}}{\sum_{k=1}^{n_{i}} v_{i k}^{\frac{1}{\alpha-2}}} .
$$

\section{References}

Akerlof RJ, Holden RT (2012) The nature of tournaments. Econ Theory 51(2):289-313

Baik KH (1993) Effort levels in contests: the public-good prize case. Econ Lett 41(4):363-367

Baik KH (2008) Contests with group-specific public-good prizes. Soc Choice Welf 30(1):103-117

Bandiera O, Barankay I, Rasul I (2011) Field experiments with firms. J Econ Perspectives 25(3):63-82

Brookins P, Lightle JP, Ryvkin D (2015) Optimal sorting in group contests with complementarities. J Econ Behav Org 112:311-323

Cheikbossian G (2012) The collective action problem: within-group cooperation and between-group competition in a repeated rent-seeking game. Games Econ Behav 74(1):68-82

Corchón LC (2007) The theory of contests: a survey. Rev Econ Des 11(2):69-100

Cornes R, Hartley R (2005) Asymmetric contests with general technologies. Econ Theory 26(4):923-946

Cubel M, Sanchez-Pages S (2014) Inequality in conflicts. Technical report, Working Paper

Dasgupta P, Sen A, Starrett D (1973) Notes on the measurement of inequality. J Econ Theory 6(2):180-187

Esteban J, Ray D (2001) Collective action and the group size paradox. Am Political Sci Rev 95(3):663-672

Fudenberg D, Tirole J (1991) Perfect bayesian equilibrium and sequential equilibrium. J Econ Theory 53(2):236-260

Hardy G, Littlewood J, Polya G (1934) Inequalities. Univ. Press, Cambridge

Marshall AW, Olkin I, Arnold BC (1979) Inequalities: theory of majorization and its applications, vol 143. Springer, Berlin

Nitzan S (1991a) Collective rent dissipation. Econ J 101(409):1522-1534

Nitzan S (1991b) Rent-seeking with non-identical sharing rules. Public Choice 71(1-2):43-50

Nitzan S, Ueda K (2011) Prize sharing in collective contests. Euro Econ Rev 55(5):678-687

Nitzan S, Ueda K (2014) Intra-group heterogeneity in collective contests. Soc Choice Welf 43(1):219-238

Nitzan S, Ueda K (2018) Selective incentives and intragroup heterogeneity in collective contests. J Public Econ Theory 20(4):477-498

Olson M (1965) The logic of collective action. Harvard University Press, Cambridge 
Ryvkin D (2011) The optimal sorting of players in contests between groups. Games Econ Behav 73(2):564-572

Ryvkin D (2013) Heterogeneity of players and aggregate effort in contests. J Econ Manag Strategy 22(4):728-743

Tullock G (1980) Efficient rent seeking. In: Buchanan JM, Tollison RD, Tullock G (eds) Toward a theory of the rent-seeking society. Texas A\&M University Press, College Station, pp 97-122

Ueda K (2002) Oligopolization in collective rent-seeking. Soc Choice Welf 19(3):613-626

Publisher's Note Springer Nature remains neutral with regard to jurisdictional claims in published maps and institutional affiliations. 